\title{
846 PRE-CLINICAL VALIDATION OF A FLT3L-FUSION PROTEIN FOR DENDRITIC CELL EXPANSION AND ANTI- TUMOR EFFICACY
}

Michelle Kuhne*, Hamlet Chu, Sarah Ng, Christopher Clarke, Brian Carr, Manuel Baca, Magdeleine Hung, Mark Nagel, Alexandre Ambrogelly, Nicholas Wilson. Gilead Sciences, San Francisco, USA

Background The ligand for the receptor tyrosine kinase FMSlike tyrosine kinase 3 (FLT3) plays an importantrole in hematopoiesis. FLT3 signaling is required for the differentiation andexpansion of dendritic cells. In the context of cancer immunity, the conventional dendritic cellsubtype 1 (cDC1) are required for the generation of tumor-specific $\mathrm{T}$ cell responses in mousepreclinical models. In human tumors $\mathrm{cDC} 1$ are often underrepresented in thetumor microenvironment, supporting the hypothesis that therapeutically increasing their number via FLT3 pathway stimulation has the potential to promote $\mathrm{T}$ cellmediated anti-tumor efficacy.

Methods GS-3583 is a fusion protein composed of the extracellular domain (ECD) of human FLT3 ligand(FLT3L) combined with a modified fragment crystallizable $(\mathrm{Fc})$ region of human IgG4. GS-3583was designed to induce cDC1 expansion and subsequently promote tumor-reactive $\mathrm{T}$ cell priming, activation and recruitment into the tumor microenvironment. To evaluate the therapeutic efficacy of FLT3 stimulation in vivo, a mouse surrogate mGS-3583was designed using the ECD of mouse FLT3L fused to an engineered mouse IgG2a Fc withattenuated binding to mouse FcgRs.

Results mGS-3583 bound to recombinant mouse FLT3 with an estimated affinity of $15 \mathrm{nM}$, and to mouse FLT3-expressing cells with an EC50 of $0.15 \mathrm{nM}$. In vivo, mGS-3583 showed single agent dose-dependent tumor growth inhibition (TGI) in tumors that correlated with peripheral and intratumoral cDC1 expansion. In tumors with no initial immune infiltration, mGS-3583 led to an influx of $\mathrm{T}$ cells into the tumors. In addition to single agent efficacy, mGS-3583 combined effectively with programmed cell death protein (ligand)1 (PD(L)-1) pathway blockade.

Conclusions In vivo expansion of dendritic cells can convert uninflamed (cold) tumors to immunologically active (hot) tumors and initiate productive anti-tumor immune responses. These findings support the development GS-3583 as a promising candidate for cancer immunotherapy.

http://dx.doi.org/10.1136/jitc-2021-SITC2021.846 